PNNL-11042

UC-810

Project Technical Information

RECFIVED

Compilation of Information on APR 011996

Modeling of Inductively Heated Cold OS.TI Crucible Melters

D. L. Lessor

March 1996

Prepared for the U.S. Department of Energy under Contract DE-AC06-76RLO 1830

Pacific Northwest National Laboratory Operated for the U.S. Department of Energy by Battelle Memorial Institute

\title{
Battelle
}


PNNL-11042

UC-810

Project Technical Information

\section{Compilation of Information on Modeling of Inductively Heated Cold Crucible Melters}

D. L. Lessor

March 1996

Prepared for

the U.S. Department of Energy

under Contract DE-AC06-76RLO 1830

Pacific Northwest National Laboratory

Richland, Washington 99352

Reprint of historical document PVTD-C95-03.01C1, Part B, transmitted with letter dated September 6, 1995. Data, formating, and other conventions reflect standards at the original date of printing. Technical peer reviews and editorial reviews may not have been performed. 


\title{
DISCLAISAER
}

This report was piepared as an account of work sponscoted by an agency of the United Staies Coleinmeni. Neither the Lnited Siaies Covernment nor any agency thereot, nor Batelle Memorial Instituie, nor any of their employees, makes any warranty, express or implied, or assumes any legal liability or responsibility for the accuracy, completeness, or, usefulness of any information, apparaius, product, or piocess disclosed, or represents that its use would not infringe privately orned rights. Reference herein io any specific commercial product, prosess, or service by irade name, bademark, menuíaciuitr, or cihenvise cues not necessarily constituie or imply its endorsement, recommendation, or favoring by the United States Covernment or any agency thereof, or Banelle Memorial Instituie. The views and opinions of authors expressed herein do not necessarily state or tellect those of the United States Government or any agency ihereof.

\author{
PACIFIC NORTHWEST NATIONAL LABORATORY \\ operated by \\ BATTELLE \\ for the \\ UNITED STATES DEPARTMENT OF ENERGY \\ under Contract DE-AC06.76RLO 1830
}

Printed in the United States of America

Available 10 DOE and DOE contractors from the

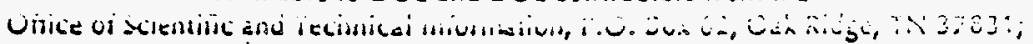
prices available from (615) $576-8401$.

Arailable to the public from the National Technical iniormation Service, U.S. Department of Commerce, 5285 Port Royal Rd., Springiteld, VA 22161 
Milestone: C95-03.01C1

Part B.

\title{
COMPILATION OF INFORMATION ON MODELING OF \\ INDUCTIVELY HEATED COLD CRUCIBLE MELTERS
}

\author{
D. L. Lessor
}

\section{INTRODUCTION}

\section{Objective}

The objective of this communication, Phase B of a two-part report, is to present information on modeling capabilities for inductively heated cold crucible melters, a concept applicable to waste immobilization. Inductively heated melters are those in which heat is generated using coils around, rather than electrodes within, the material to be heated. Cold crucible or skull melters are those in which the melted material is confined within unmelted material of the same composition.

This phase of the report complements and supplements Phase $A$ by Loren Eyler, specifically by giving additional information on modeling capabilities for the inductively heated melter concept. Eyler discussed electrically heated melter modeling capabilities, emphasizing heating by electrodes within the melt or on crucible walls. Eyler also discussed requirements and resources for the computational fluid dynamics, heat flow, radiation effects, and boundary conditions in melter modeling; the reader is referred to Eyler's discussion of these. This report is intended for use in the High Level Waste (HLW) melter program at Hanford. We sought any modeling capabilities useful to the HLW program, whether through contracted research, code license for operation by Department of Energy laboratories, or existing codes and modeling expertise within DOE. 


\section{Approach}

The assessment of computational codes, modeling capabilities, and modeling needs included the following activities:

1. A literature search was performed, seeking reported models of inductive heating for either glass melting or crystal production.

2. Papers on inductive heating for glass processing and crystal growth and papers from the cold crucible melter laboratories and vendors were studied.

3. A concept for the linking of a computational fluid dynamics (CFD) calculation to an inductive heat source computation for cold crucible melter simulations was developed, and a hypothesized list of phenomena one might want to elucidate by modeling was drawn up. This concept served in guiding questions to vendors.

4. A number of firms and laboratories involved in the inductively heated cold crucible melter concept were contacted and asked about models used by or available to them, and their availability to us.

5. A number of vendors of commercial computational fluid dynamics (CFD) computer codes were called, seeking their knowledge of applications by their clients of their codes to inductive heating.

6. Electromagnetic field codes suitable for linking to CFD codes to calculate inductively generated heat were discussed with contacts at other laboratories and at code vendors. A search for U.S. government owned computer codes for electromagnetic fields computations in the Energy Science and Technology Software database, formerly the National Energy Software Center, was performed. The Los Alamos Accelerator Group Codes Compendium was studied for codes relevant to electromagnetic fields modeling.

The information found and conclusions drawn from these activities are summarized in this report. 


\section{Modeling Requirements and Model Features}

Purpose

This section sets forth the hypothesized modeling requirements and some model features to study treatment of Hanford wastes with inductively. heated cold crucible melters. The hypothesized model features and requirements are based on previous work with electrode-heated melters and on reports in the literature on phenomena and models for inductively heated melters for glass or large crystal production.

Information and Decision Guidance Sought from Modeling

We would like to learn:

1. Transient information:

- Melter start-up

- Solid-melt interface migration

- Melting rates

- Evolution of temperature distribution and convection cells

- Control stability (sensitivity of quasi-steady state to power or other parameters)

2. Residence time requirements and implied maximum melter throughput for a given geometry, power level, and waste-frit combination, as determined by

- Penetration and energy deposition profile

- Heating and flow transient

- Temperature distribution

- Radio frequency driving characteristics and resulting heat deposition profile

- Velocities and shapes of convection cells

- Melting and mixing rates

- Uniformity requirements on particulate melting

- Bubble clearing rates

- Bubble removal requirements

- Bubble injection or mechanical agitation

- Physical and chemical form of the waste and frit 
(Note that many of the pieces of information would be obtained by applying correlations, back-of-envelope computations, and engineering judgement to the results of computer simulations, or by comparing simulations. For example, no information about bubble removal requirements or uniformity requirements for particle melting need be built into codes.)

3. Energy requirements and energy balance information:

- Heat delivered to vessel coolant and to batch

- Radiative heat losses

- Sensitivity of energy flows to solid-melt interface location

- Impedance and energy coupling characteristics during transient

- Information for optimizing vessel sector geometry for energy transmission considerations

\section{Inputs for Simulations}

It is assumed that inputs for simulation will include:

- Geometry of vessel and contents at start of simulation

- Initial temperature distribution

- Thermophysical properties (density, enthalpy or specific heat, thermal conductivity, viscosity of melt) of batch and structures

- Electrical properties (conductivity and dielectric constant as function of temperature at desired frequency) for batch and structures

- Coil geometry, driving current or voltage amplitudes, and frequency for electrical driving

Assumptions

Some assumptions which we consider to be valid include:

- The processes of greatest interest can be modeled by a coupled calculation of fluid dynamics, conductive heat flow, surface radiation and convection, and volumetric inductively produced heat.

- Modeling the contraction on melting can be avoided.

- Energy transport by radiation within the melt is adequately represented by an effective thermal conductivity model.

- Density is a linear function of temperature, so the Boussinesq 
approximation for buoyancy effects is valid.

- Chemical reactions and heats of fusion need not be modeled or can be modeled in a simplified way.

- Electrical properties are obtainable functions of temperature.

- Three-dimensional models for electromagnetic. fields will be needed, unless it can be shown that departures from rotational symmetry by such features as containment vessel sectors can always be accounted for by prescriptions in a two-dimensional treatment for the configurations of interest.

- Three-dimensional models for heating and fluid flow will be needed, unless it can be shown that departures from rotational (or other) symmetry will be negligible for all configurations of interest.

- The critical features needed for the fluid dynamics and heat flow computation are the ability to compute flows of extremely viscous liquids with strongly temperature-dependent viscosities, the ability to model the transition from solid to liquid in the melt process, and the ability to judiciously recompute the heat source when and only when conditions have altered it.

- The critical feature of the electromagnetic fields code is the ability to generate the current distribution in the melt region where the attenuation length is on the order of a meter, modeling adequately the effects of the metal crucible segments in which the attenuation length is on the order of 10-4 meter, at a frequency for which the vacuum wavelength is 1000 meters, in the presence of structures whose dimensions are on the order of 10-2 meter. Either a combination of volumetric and surface elements, or the ability to handle elements of greatly different size scales, will be needed.

- A critical feature of a merged computations code is the ability to predict scale-up effects, which depend intricately on electromagnetic field penetration, energy deposition profiles, and both conductive and advective heat transport

Other features of a satisfactory computational fluid dynamics (CFD) code, such as boundary condition imposition and ability to model liquids in contact with thermally conducting solids, were discussed by Eyler and are applicable here. We emphasize here the features which we consider to be key criteria for inductively heated melters: viscous flow treatment, solid-to-liquid transition, and ability to effectively use different time 
scales of different processes (convective flow, heating, melt front motion) for efficient computation.

\section{Model Concept}

The computational model which we envision as satisfying the simulation requirements described above has the following features: 1) transient computations of heat and fluid flows, with robustness for computation of highly viscous flows, and with temperature-dependent viscosity and other properties; 2) a term for heat generation per unit volume (or per computational cell) in the energy equation for the heat conduction and fluid dynamics calculations; 3) electromagnetic fields calculations to compute the volumetric heat generation by eddy currents, properly incorporating effects of crucible segments; 4) a computation of the electrical conductivity as a function of temperature, so that the spatial dependence of the electrical conductivity can be supplied at any time step from the fluid dynamics calculations to the electromagnetics calculations; 5) logic and testing within the fluid dynamics calculation to determine when in the transient simulation a recalculation of the heat generation is needed; 6) a procedure in the computations for transitions from solid to liquid with melting.

\section{CONCLUSIONS AND STRATEGY RECOMMENDATIONS}

Several conclusions can be drawn about modeling of inductively heated melters for Hanford waste immobilization and about the computational tools available to do it.

\section{Conclusions}

- Modeling is extremely important and useful as an engineering tool for inductively heated melter design, because many design questions (geometry, frequency, energy balance, scale-up, control stability) are best answered by a model. For example, optimization of the crucible segmentation would be difficult and costly without a computational code.

- While modeling attempts and experience have been limited, attempts 
reported in the literature on modeling inductive heating for glass melting and crystal growth suggest that it can be done very successfully.

- Most modeling attempts on inductively heated glass melters have modeled heat flow but not fluid flow.

The modeling performed by French organizations interacting with or coordinated by Commissariat a l'Energie Atomique (CEA) is probably good and indicative of the tractability and power of modeling of the cold crucible melter concept, but the proprietary nature of these models may make it difficult for DOE to use them in independent and objective evaluations, as cautioned by Antoine Jouan of CEA. The modeling work falls somewhat short of the objectives stated above, e.g., it has not successfully predicted the location of the melt front. Also, heat flow is modeled but fluid flow is not, to the best of our ability to determine the nature of their proprietary tools.

Models of inductive melting and recrystallization in attempts to produce large high quality crystals has been reported by a number of groups. (See, for example: Brandon, S.; Derby, J.J.; Atherton, L.J.; Roberts, D.H.; Vital, R.L.; Journal of Crystal Growth, vol.132, no.1-2 p.261-79, reporting work from the Department of Chemical Engineering \& Material Science, Minnesota University, Minneapolis, Minnesota; or Gresho, P.M.; and Derby, J.J.; Journal of Crystal Growth, vol.85, no.1-2, p.40-8, reporting work from Lawrence Livermore National Laboratory, Livermore, CA). The models have some of the features desired in an inductively heated glass melter model: inductive heating, solid to liquid or liquid to solid phase changes, fluid flow. These successful inductive crystal melting and recrystallization simulations further support the feasibility and fruitfulness of modeling for inductively heated waste in glass. Reported models were either two-dimensional or they did not include fluid flow. None appear directly applicable without modification to inductively heated glass melters for waste immobilization.

- Battelle Frankfort, which has extensive experience in simulations of 
waste melters with electrode heating, has compiled information on modeling capabilities world wide for induction melting, and is about to launch into development of an induction and microwave melting code. Ashok Rastogi at Battelle Frankfort has selected the MAFIA (MAxwell's equations using Finite Integration Algorithm) electromagnetic fields code for linking to their BASSIM (Glass version) code for computational fluid dynamics (CFD). BASSIM's capabilities are somewhat similar to those of TEMPEST, with one added feature of non-orthogonal body-fitted coordinates in its finite volumes grid, while TEMPEST has orthogonal curvilinear coordinates, including body-fitted. BASSIM author Ashok Rastogi reports that a user manual in English is available for BASSIM, which is written in FORTRAN. Rastogi has invited Pacific Northwest Laboratory (PNL) to participate in this code development effort, which aims to develop a code to be marketed jointly by CST/University of Darmstadt and Battelle Frankfort. CST is one of two firms marketing MAFIA; the University of Darmstadt holds patent rights. No PNL commitment has been made. The resulting code, with or without PNL participation, may eventually be available for contracted simulations. It is significant that Battelle Frankfort has surveyed available codes and simulations, and has elected to develop a new one. A key deficiency in previous work which led to this Battelle Frankfort decision was the failure of previous glass melter simulations to include fluid flow effects. Rastogi points to the compatibility of BASSIM's finite volumes and MAFIA's finite differences grids as a strong point for this particular linkage.

A strategy which takes advantage of DOE investments in code development, code validation, and melter simulation experience is the linking of TEMPEST (a PNL code) for heat and fluid dynamics calculations with the TORO II code for electromagnetic field computations. TEMPEST, developed at Pacific Northwest Laboratory, has a history of successful application to glass waste melter problems, while TORO II, developed at Sandia National Laboratories, is in the final phases of validation and documentation. Because both are DOE codes, a linked code would be available for use throughout . DOE. TORO $I I$ is a finite elements code, which is appropriate for modeling electromagnetic fields in a region whose subregions have 
vastly different attenuation lengths. TORO $\mathrm{Il}$ is applicable to radio frequency heating (where the vacuum wavelength is long compared to modeling region dimensions), but not to microwave heating, where many wavelengths can fit within the modeled regions. Some simplification of boundary conditions result from abandoning applicability to microwave frequencies. The transfer of temperature and heat source information between TEMPEST's finite volumes representation and TORO Il's finite elements using an interpolation scheme is expected to present no problems. Finite volume codes have been found by some to maintain continuity more accurately in fluid flows than finite elements codes, so finite volume codes may be preferable for the fluid dynamics calculations. Because both TEMPEST and TORO II are DOE codes, access to source code would allow efficient transfer of information and permit code modifications as needed to handle the liquid-to-solid transition. Also, internal decision making on such questions as when to update the heat source term is a strong point of TEMPEST which would be utilized.

- Another source of electromagnetic fields code expertise within DOE is the Electronics Engineering Department of the Engineering Research Division of Lawrence Livermore National Laboratory. Unfortunately, a search of their developed codes described on their World Wide Web pages finds none immediately applicable to inductive melter heating without code development work. Clifford Shang has indicated an interest in adapting existing codes to the inductive melter needs, but their surface integral formulation codes seem an inappropriate starting point. Finite element and finite differences codes are probably better.

- Other linkages of commercial codes with these DOE codes or with each other are possible, and a satisfactory code for inductively heated melter studies could probably be developed. Lack of access to source code in the commercial codes would be an inconvenience, though not prohibitive. Licensing restrictions would limit transferability between laboratories within the DOE system.

- Much other electromagnetic fields code work in DOE is focused on 
the scattering problem, and is not applicable to melters. However, a 2-dimensional electromagnetic field eddy current computation was part of a crystal growth simulation at Lawrence Livermore Laboratory. Eddy current calculations for systems having rotational symmetry and only azimuthal direction currents are particularly simple but not trivially extended to three dimensions.

The ARGUS code, developed by SAIC (Sciençe Applications International Corporation, 1710 Goodridge Drive, McLean, Virginia 22101), like the commercially available MAFIA code, is a massive code with much charged particle trajectory calculation capability not needed for inductively heated melter simulation, which we judge would make it cumbersome to link to quickly to a computational fluid dynamics code. A joint effort with the code proprietors involving access to source code would probably be needed.

- A number of commercial electromagnetic field codes which appear suitable for calculating inductive heating are available. Lease costs are typically on the order of $\$ 10 \mathrm{~K}$ to $\$ 25 \mathrm{~K}$ per year, or on the order of $\$ 35 \mathrm{~K}$ to $\$ 60 \mathrm{~K}$ for an indefinite lease of a 3-dimensional code. Codes available include: Maxwell 3D from Ansoft Corporation; the MagNet system, particularly the TH-3D solver (for Time Harmonic 3Dimensional), from Infolytica Corporation of Montreal; EMAS from MacNeal-Schwendler; MAFIA from U. S. sales representative AET Associates, Inc., for the University of Darmstadt; ELECTRA from Vector Fields, Inc. These are all established codes used by large numbers of licensees. Unfortunately, they are very large (some exceeding one million lines of coding), and consequently require a large investment in user familiarization, especially for linking to other codes.

A number of commercial computational fluid dynamics codes are available. Among the best known are FIDAP, FLUENT, and Flow-3D. The latter is available at PNL. Capabilities are probably good, and provision is made for user routines for such things as heat sources. Vendors point to applications by their clients to glass melting, though not by induction. We found no interest among the vendors for adding inductive electrical heating as a capability. Lack of access 
to source code is expected to be some (though surmountable) inconvenience when others attempt to add inductive heating features.

- Some electromagnetic fields computational capability is now available with the widely used ANSYS code, already leased by PNL. Interestingly, some flow computation ability has recently been added to the ANSYS package when ANSYS owners purchased COMPUFLO, which owns rights to the FLOTRAN computational fluid dynamics code. The degree of compatibility of these capabilities and the appropriateness to inductive melter modeling is untested, however.

\section{Recommendations}

A linkup of a computational fluid dynamics code and an appropriate electromagnetic fields code should be performed as a valuable and cost effective step in evaluating and guiding development of the inductively heated melter concept. The linking of the TORO II code with the TEMPEST code seems to be a good choice for a modeling tool for inductively heated melters. Reasons include:

- Both are DOE codes, unhampered by license agreements, and available without substantial additional cost.

- TEMPEST has a record of success in modeling flow in glass melters.

- TORO II seems to have the needed features and to be free of unneeded features for radio frequency glass melting, to a greater extent than any other code considered.

If it is deemed absolutely essential to be able to model radio frequency heating and microwave startup with the same code, or if TORO II proves unsatisfactory or unavailable for any reason, then a commercial code (possibly Maxwell 3D from Ansoft, the ELECTRA code from Vector Fields, Inc., the MagNet codes from Infolytica, the ARGUS code from SAIC, or the MAFIA code) should be used instead of TORO II. We consider this extension to include microwave heating in addition to radio frequency heating to be a significant additional step that probably more than doubles the labor and 
complexity of the linkup.

The code which Battelle Frankfort proposes to develop should be considered as a backup to the DOE code whose development we are suggesting, but we believe that a more open evaluation, discussion of assumptions, and presentation of results will come from a domestic DOE activity. The code suggested here, assuming use of TEMPEST and TORO II, should be the simpler one to use for radio frequency melter simulations.

\section{Institutions With Inductive Glass Melting Capability}

\section{VECTRA EnviroGlass}

\section{P. O. Box 1164}

2939 Richardson Road

Richland, WA 99352

(509)375-3320 Fax (509)375-6225.

\section{Contact: Bruce Mason, P. E., Chief Engineer}

VECTRA has contracted a vendor to provide to VECTRA code capabilities for induction heated cold crucible melter modeling. VECTRA will not reveal the name of the vendor, and considers the product code and the vendor name proprietary. However, VECTRA did supply a one page description of the code. It is our impression that the vendor is probably allowed to provide computation services to other customers than VECTRA, but the customer would need to find out who the vendor is. VECTRA hopes to recover their costs in the code capabilities contracted from the vendor through contracts for waste melter research and development.

The code uses the EMAS finite element program for electromagnetic field computations, and the finite element ANSYS program for the computation of thermal effects. EMAS is a product of the MacNeal-Schwendler Corporation; and uses the same solver as MacNeal-Schwendler's upgraded version of the NASTRAN program. From information supplied by Vectra, we conclude that the computed thermal effects include temperatures based on thermal conduction and resulting stresses, but do not include flow effects. The software was reported as running on a Hewlett-Packard workstation equipped with $64 \mathrm{MB}$ of RAM and $2.8 \mathrm{~GB}$ of hard drive storage, 
allowing fairly large problems to be solved:

EMAS solves Maxwell's equations in two or three dimensions in either time domain or frequency domain, the latter being the form of interest for high frequency melter simulations. Eddy current effects, the primary heating mechanism, are accounted for. Heat generation in crucibles, loads, and other components has been simulated. Simulations of a sectioned crucible "pie slice" have been performed.

EMAS and ANSYS have been used together by the vendor to simulate thermal effects from induced currents in an induction graphitizing furnace. We were given the impression that VECTRA had utilized the code capabilities in seeking DOE melter contracts.

\section{Commissariat a l'Energie Atomique (CEA)}

CEA Centre d'Etudes Nucleaires de la Vallee du Rhone 30-Bagnols-sur-Ceze (France)

\section{Contact: Antoine Jouan}

Phone: 011 3366796376

French organizations (CFEI, CEA, ELECTRICITIE DE FRANCE, MADYLAM Laboratory) involved in inductively coupled cold crucible melter work appear to rely on a 3-D model developed jointly by the MADYLAM laboratory and CEA. The CEA model is essentially the code from MADYLAM. This code, known as MALICE, uses the 3D electromagnetic package OPHELIE, and solves the thermal effects equations with finite elements partial differential equation solver FLUX EXPERT. The solution technique for the electromagnetic part is described as the integral method. From a 1992 paper from MADYLAM published in Journal de Physique III France 2 (1992), p1991-2004, we see an energy transport term from flow in a steady state energy equation, but no indication that a flow equation was solved. The modeling in MALICE is thus less sophisticated than our "conceptual model" presented earlier.

Successes with the MALICE computation include predictions of the locations of the heat generation and optimization of the number of crucible sectors. Antoine Jouan reports, however, that they have not been successful in predicting the location of the melt front. We suspect that 
computing flow patterns is necessary to achieve that.

GOI im. Vavilova (Approximately "S. I. Vavilov State Optical Institute")

Russia

Publication title: Mathematic Model of Mass and Heat Transfer in Glass Melt in a Cylindrical Induction Furnace

AUTHOR(S): Zhilin, A. A.; Zamytin, A. N.; Shiff, V. K. Work published in: Steklo I Keramika ("Glass and Ceramics"), no. 3-4, p. 19-22, 1994

We learned little of this work, aside from abstract keywords. It was published in Russian, though a translation may be available as "Glass and Ceramics." We have requested this translation. We do not know the level of sophistication of the modeling work, but we mention it here because it is the only published work we found in the literature search which apparently included flow and not just heat conduction in the model for an inductively heated glass (as opposed to crystal or metal) melter. Cylindrical symmetry was assumed.

Electromagnetic Fields Codes Organizations

Ansoft Corporation

Four Station Square, Suite 660

Pittsburgh, PA 15219

Phone: (412)-261-3200 Fax: (412) 471-9427

Contact: Hank Campbell

Ansoft Corporation licenses a number of electric and magnetic field codes to users. Maxwell 3D is their electromagnetic field code which would be a candidate for linkup for inductive melter simulations. From promotional information sent to us, a number of highlighted features are particularly relevant to inductive melter modeling:

- $\quad 3 D$ Eddy Current solver for $A C$ field solutions, including skin and proximity effects

- Uses finite elements analysis

- Small skin depths can be modeled efficiently using a surface- 
impedance boundary

- Automatic Delaunay mesh generation ensures optimal mesh for given model

- Automatic refinement of mesh based on accuracy analysis

- Manual refinement of mesh available in cases where critical areas are already known

- Second-order elements greatly increase accuracy of internal interpolations

- Complete field solutions of V, E, D, J, B, and $H(V=$ voltage field, $E=$ electric field vector, $D=e l e c t r i c a l$ displacement vector field, $J=$ charge flux field, $B=$ magnetic induction, $H=$ magnetic field vector)

- Automatic computation of impedance, ohmic losses, hysteresis loss

These features appear to give Maxwell 3D very good capabilities for the electromagnetic fields computations in inductive melter modeling. Difficulties in achieving the required information transfers between the computational fluid dynamics code and Maxwell 3D are possible, though probably minor and solvable. We found no interest from Ansoft in linking their code to a computational fluid dynamics code themselves, though they would provide the usual technical support to a licensee who wished to do so.

Cost and the limitations of the licensing agreement are significant drawbacks. Maxwell 3D is available on a perpetual license only. For a UNIX workstation, the license price is $\$ 34,900$. Broad capabilities of Maxwell 3D also mean that a fair amount of effort would need to go into learning how to use it.

\section{Infolytica Corporation}

P.O. Box 1144, Stn. Place du Parc,

300 Leo Parisseau, Suite 2222

Montreal, Canada H2W 2P4

Phone: (514)849-8752

Contacts:Herman Vanegas Ext. 257

Bizat Forgani Ext. 210

Infolytica Corporation licenses the MagNet family of electric and magnetic field codes and modules. Infolytica's TH-3D (for Time Harmonic, 
3 Dimensions) solver is the heart of the package that would be applicable to the inductively heated melter simulation. The TH-3D eddy current solver uses a mix of scalar and vector potential in its finite elements analysis. This is not the covariant 4-vector potential, however. Infolytica claims a reduction of up to a factor of 10 in computing times from using this mix of scalar and vector potentials. A line of pre- and post-processors is available, along with modules to meet the needs of specific customers. We supplied Infolytica technical staff with an. inductive melter schematic for their comments. They suggested for our modeling requirements the MagNet TB-2D/3D TH (time harmonic) module, the Loss2D/3D modules for the computation of eddy current heating, and the Large and Huge solvers, to give us a mesh capability up to 217,000 bricks. A few other items relevant to applicability of the MagNet family of modules to inductive melter applications:

- The MagNet family's mesh generation takes into account only input geometry, not electrical conductivity, in selecting the mesh. Adaptation to provide the accuracy needed is provided by varying the order of the of the finite element representation, with orders from first to third possibly present at different locations in the same simulation.

- Impedance boundary conditions at surfaces, which would be useful at the surfaces of high conductivity regions like metal crucible segments, are not yet available, though they hope to add this feature in the future. Surface impedance boundary conditions allow bypassing multilayer representation of the attenuation region at the surface of high conductivity regions.

- Infolytica technical staff thought that their adaptive order for 3dimensional elements would allow representing the metal crucible sector skins with only two layers of elements. (By contrast, Cliff Shang at Lawrence Livermore National Laboratory thought ten layers would be needed to characterize the skin depth attenuation, but codes from LLNL's EIGER project bypass this need with surface impedance boundary conditions. Unfortunately, we learned of no LLNL codes which treat the volumetric conduction effects.)

- Infolytica's eddy current computation has been previously linked to a heat flow computation, and they have modules that they would supply to a licensee to expedite a comparable linking to fluid flow. 
Their eddy current codes will write a file of heat generation per unit volume at a user-supplied list of points. That list of points could be the cell centers in a computational fluid dynamics simulation.

- Infolytica expressed no interest in doing the linking of their electromagnetic fields codes to computational fluid dynamics codes themselves.

Prices for the code package which Infolytica recommends for our application are:

One year license

Indefinite license
$\$ 21,250$

$\$ 51,250$

The MacNeal-Schwendler Corporation Electromagnetics Application Department 9076 North Deerbrook Trail Milwaukee, Wisconsin 53223 PHONE: 1 (414) 357-8723 Contacts:David Cole, National Sales Manager for Electromagnetics Software. (714)444-5199. Patrick Wallen, Technical Specialist in Electromagnetics. (714)444-5198

The MacNeal-Schwendler Corporation licenses the NASTRAN finite elements codes for stress and heat flows, and offers the EMAS program based on a similar solver for electromagnetic fields computations. Some of the information here comes from the Los Alamos Accelerator Group Code Compendium, in addition to MacNeal-Schwendler staff.

MSC/EMAS is an Electro Magnetic Analysis System. It is a general-purpose two- and three-dimensional finite-element analysis program for electromagnetic problems with linear, nonlinear, and anisotropic materials. It includes DC, AC, transient, and modal analysis. The user can mix three-, two-; and one-dimensional finite elements plus circuit elements to model sources. The two-dimensional elements should be 
useful in modeling surfaces of high-conductivity regions without resolving detail of attenuation within a skin depth of the surface. The program includes a graphics preprocessor and postprocessor. Development has been under way since 1967. The first-release of MSC/EMAS came out in October 1989. It has approximately one million lines of FORTRAN-77 source code and some (approximately 2\%) Assembler. Source code is not available to licensees. An approximately 1000-page user's guide, an approximately 200-page application manual, and examples are available, as are courses for users.

Patrick Wallen was aware of much work in which EMAS had been linked to a NASTRAN structural mechanics and heat flow computation for devices like transformers, but he was unaware of linkages of EMAS to computational fluid dynamics. He noted that MacNeal-Schwendler has a fluid dynamics code, MSC-DYTRAN, a finite volumes code which can do compressible fluid flows, but this is beyond the needs and anticipated budgets for inductively heated melter modeling. Both workstation and supercomputer codes can be licensed on a monthly basis, and workstation code can be purchased. Patrick Wallen estimated that a 10,000 element mesh representation of the inductively heated melter electromagnetic fields could be run in 15 minutes on a Sparcstation 20 . We anticipate on the order of 20 calls to an electromagnetic fields calculation in an inductive melter simulation. David Cole suggested that minimal workstation requirements are a $125 \mathrm{MHz}$ processor, $128 \mathrm{Mb}$ of RAM, and 2 to $4 \mathrm{~Gb}$ of disk.

We suspect that EMAS can do the electromagnetic field calculations needed for inductively heated melter modeling, as evidenced by its application by a vendor in code developed for Vectra Enviroglass, but that its huge size and generality makes it unnecessarily difficult for this application. Todd Hubing, an EMAS user from the University of MissouriRolla's Electromagnetic Compatibility Laboratory and Electrical Engineering Department, confirms that a fair amount of effort is needed to learn to use EMAS.

Prices quoted for EMAS for a workstation are:

Purchase: $\quad \$ 30 \mathrm{~K}$ to $\$ 60 \mathrm{~K}$ (depending on modules) 
Lease:

$\$ 1200 /$ month to $\$ 2000 /$ month.

Technische Hochschule Darmstadt (University of Darmstadt)

Thomas Weiland ( senior MAFIA code author)

Institut für Hochfrequenztechnik

Fachgebiet Theorie Elektromagnetischer Felder

Schlossgartenstrasse 8

D-6100 Darmstadt

Federal Republic of Germany

U.S. Distributor:

AET Associates Inc.

20370 Town Center Lane

Suite 260

Cupertino, California

Phone: (408) 9961760

Fax: (408) 9961962

The MAFIA code was produced by a collaboration among Technische Hochschule, Darmstadt, Federal Republic of Germany; Deutsches

Elecktronen-Synchrotron (DESY), Federal Republic of Germany;

Kernforschungs Anlage-Jülich (KFA), Federal Republic of Germany; and Los Alamos National Laboratory. Thomas Weiland, Technische Hochschule, is credited as the principal author. The information here was taken from the Los Alamos Accelerator Group Code Compendium, from code manuals available from the National Energy Research Supercomputer Center (NERSC), from Ashok Rastogi of Battelle Frankfort, and from AET Associates, Inc. The MAFIA code is available for a fee in the U.S. from AET Associates, Inc., (408) 996-1760. A call to AET Associates resulted in the sending of a catalog, which included a form for requesting a price quote, but no pricing information.

MAFIA ( MAxwell's equations solved by the Finite Integration Algorithm) is a family of codes that solves Maxwell's equations for static, resonant, and transient fields. The mesh generator, postprocessor, and the units that handle the physics are separate modules within the family of codes. Extensive particle trajectory computation (particle in cell) capabilities 
are also available for accelerator design studies. For inductive heating computations, the W3 eddy current module, along with the pre- and postprocessor models would be needed. MAFIA had approximately 83,500 lines of FORTRAN source code as installed at Los Alamos National Laboratory and at the National Energy Research Supercomputer Center (NERSC) at Lawrence Livermore National Laboratory in 1990, and has probably grown a fair amount (We estimate by a factor of 10 ) since then. MAFIA is a finite differences rather than a finite elements code. Sixteen megabytes memory is required for the August 1995 version.

Because of the size and complexity of the code, a fair amount of labor would be involved in the linking to computational fluid dynamics codes for inductively heated melter modeling.

\section{University of Missouri-Rolla}

Electrical Engineering Department

Electromagnetic Compatibility Laboratory

Contact: Todd Hubing (314) 341-4506

The Electromagnetic Compatibility Laboratory makes available the EMAP (ElectroMagnetic Analysis Program) family of codes as public domain. Because these codes do not model lossy dielectrics or conducting materials, they are not appropriate to inductive heating. Hubing has used the EMAS code from MacNeal-Schwendler and is familiar with the Maxwell 3D code of Ansoft Corporation. He cautioned that both require a massive effort to learn to use. Typically, an organization licensing them has a person dedicated to knowing the capabilities and modeling techniques. Hubing confirmed that both EMAS and Maxwell 3D have the capabilities for inductive heating in melters.

Vector Fields, Inc.

1700 North Farnsworth Av

Aurora, IL 60505

Telephone: (708)851-1734

Fax: $\quad(708) 851-2106$

Contact: David C. Carpenter, Vice President

ELECTRA is Vector Fields' computational code for AC and transient 
analysis, which they report has been used by some customers to model inductive heating. OPERA-3d is their 3-dimensional pre- and postprocessor. Use of their $3 d$ package for inductive heat calculation linked to a 3d thermal (but not fluid flow) computation is reported by Carpenter to have been done by SDRC, a Milford, Ohio-based engineering services firm. The following is a quote from Vector Fields' brochures:

The ELECTRA analysis package is a module of the OPERA-3d integrated suite of finite element software for 3D electromagnetic design analysis and simulation. ELECTRA computes time varying fields, both time harmonic and transient in three dimensions and is well proven over many years of industrial use. ELECTRA is a second generation package using advanced numerical methods for accuracy and speed of computation.

ELECTRA has the following features:

- Full 3D modeling

- Efficient Data Input

- Time harmonic field analysis

- Transient field analysis

- High speed motion analysis

- Nonlinear materials

- Complex permeability

- Hysteresis effects

- Interfaces to CAD/CAM

- Extendible Post Processing

ELECTRA is a finite element code, and is reported by Vector Fields to have been used to calculate eddy current heating (along with many other uses). The numerical techniques in ELECTRA are also discussed in their brochure:

ELECTRA uses specialist finite element analysis techniques for time varying electromagnetic fields. The time variation can be time harmonic (steady state $A C$ ) or transient. In addition the effects due to high speed motion can be computed. The ELECTRA method uses the total and reduced magnetic scalar potentials in non conducting media thereby correcting the cancellation errors associated with the simple reduced potential approach, and reducing the computation 
time. In conducting media the program uses the magnetic vector and electric scalar potentials which are directly coupled with the potentials in the exterior. This enables different conductors in contact (including holes) to be modelled accurately. To enable the effects of high speed motion to be modelled ELECTRA uses a numerical technique known as 'upwinding.'

We conclude that the numerical methods are similar to those in Infolytica's MagNet codes. Some features are unneeded for inductively heated melter modeling, of course. For example, we need only harmonic, not transient, analysis, and we do not need high speed motion effects. On a Unix workstation, ELECTRA with OPERA-3d requires $8 \mathrm{Mb}$ minimum (16 $\mathrm{Mb}$ preferred) memory. and $320 \mathrm{Mb}$ hard disk.

Vector Fields offers a number of license options and package combinations. One probably adequate for inductively heated melter simulations is:

Pre and Post Processors and 3 Analysis Packages...

1 year lease

Indefinite lease
$\$ 20 K$

$\$ 36 \mathrm{~K}$

\section{Sandia National Laboratories}

\section{Engineering Sciences Center}

\section{Albuquerque, New Mexico 87185}

Contact: David K. Gartling, (505)844-9150

David Gartling is in the final stages of documenting the TORO II code, a finite elements electromagnetic fields code developed for coupling to applied mechanics codes for stress, fluid flow, or heat flow. Gartling has supplied us with a draft of two TORO II manuals, a theory manual and a user's manual. TORO II neglects the displacement current as small compared with conduction current, which is a very useful simplification valid in the frequency domain in which the modeled region is small compared with the vacuum wavelength (1000 meters at $300 \mathrm{kHz})$ and the following condition in rationalized MKS (meter-kilogram-second) electrical units holds: dielectric constant $x$ permittivity of vacuum $x$ frequency $\times 2 \pi$ « electrical conductivity, where the permittivity of vacuum is $8.85 \times 10^{-12} \mathrm{Farad} / \mathrm{m}$. This simplifies numerics and boundary conditions. Gartling has indicated interest in having this DOE code applied 
to inductively heated melter simulation. At Gartling's request, we supplied a line drawing and some specifications for possible use as an example in one of the forthcoming TORO manuals.

The following is taken from the TORO-ll draft theory manual:

The TORO-II computer code is a general purpose program designed for the solution of static and quasi-static problems involving electromagnetic fields. The code is based on the Galerkin form of the finite element method (FEM). The present version of TORO II represents a significantly extended edition of the original program which was limited to axisymmetric geometries.

The class of problems recognized by TORO II include the scalar potential problems associated with electrostatics and steady current flows as well as the vector potential problems of magnetostatics and eddy currents. These types of field problems are readily simulated in arbitrary two-dimensional and threedimensional geometries. Derived electric and magnetic field variables are also provided by TORO II as are the thermo-mechanical quantities, such as Joule heating and Lorentz forces. The coupling of TORO II with other mechanics codes was a primary consideration in the code design.

A significant effort has been made during the development of TORO II to create an analysis program that is easy to use.

Pre-processors, including mesh generation, and post-processors for TORO II are being documented by Gartling.

TORO II appears to be best suited of any of the electromagnetic fields codes located for linking with computational fluid dynamics codes in a powerful, comparatively simple, portable computational tool for inductive melter simulations within the DOE laboratory system.

\section{Lawrence Livermore National Laboratory Electronics Engineering Department}


Livermore CA

Computational Electronics and Electromagnetics Thrust Area Clifford C. Shang, Thrust Area Leader

The EIGER project, an activity involving participants from Lawrence Livermore National Laboratory, Sandia National Laboratories, University of Houston, and Navy Command and Control.Ocean Surveillance Center, seeks to produce a code of much generality, but its capability currently extends to scattering and not to inductive melter heating. From the descriptions of this group's codes on the World Wide Web pages, none is currently satisfactory for inductive melter simulations; most are appropriate for electromagnetic wave scattering from surfaces.

As described on their World Wide Web pages, the ElGER code has surface elements characterized by surface impedance, but does not have volumetric elements with finite conductivity. After what seems to us as a fairly major addition, i.e., volumetric and surface elements in the same code, it is possible that codes from the EIGER program for electromagnetic fields computations would permit calculation of both startup microwave heating and the subsequent transient with radio frequency heating as the heat source. Somewhat more difficult boundary conditionis for the user to supply is expected to accompany this capability for microwaves and radio frequency, compared with a code designed for radio frequency eddy current (no microwave, no radiation) computations alone. Impedance boundary conditions bypass nodalization problems for the near-surface region of high conductivity regions with a small skin depth. Impedance boundary conditions, intelligent automated nodalization, or intelligent choice of order in finite elements are approaches to handling these near-surface problems in high conductivity regions, but one must also have the volumetric elements to describe Joule heating in the glass.

Management of Lawrence Livermore Laboratory's Computational Electronics and Electromagnetics Thrust Area has indicated some willingness to adapt a version of one of their codes for use in the inductively heated melter studies. It is probably better, however, to start from a finite elements, finite differences, or finite volumes code and to add the impedance boundary conditions, than to start with a surface 
integral code and try to add volumetric elements. Hence we do not rate highly the development of needed codes from Lawrence Livermore's existing codes. 